\title{
Gold Nanoparticles Modified a Multimode Clad-Free Fiber for Ultrasensitive Detection of Bovine Serum Albumin
}

\author{
Vu Thi Huong, ${ }^{1,2}$ Nguyen Tran Truc Phuong, ${ }^{1,2}$ Nguyen Tien Tai, ${ }^{1,2}$ Nguyen Thuy An, ${ }^{1,2}$ \\ Vu Dinh Lam, ${ }^{3}$ Do Hung Manh, ${ }^{4}$ Tran Thi Kim Chi, ${ }^{4}$ Ngoc Xuan Dat Mai, ${ }^{2,5}$ \\ Viet-Duc Phung $\mathbb{1},{ }^{6,7}$ and Nhu Hoa Thi Tran $\oplus^{1,2}$ \\ ${ }^{1}$ Faculty of Materials Science and Technology, University of Science, Ho Chi Minh City, Vietnam \\ ${ }^{2}$ Vietnam National University Ho Chi Minh City, Vietnam \\ ${ }^{3}$ Graduate University of Science and Technology, Vietnam Academy of Science and Technology, Hanoi, Vietnam \\ ${ }^{4}$ Institute of Materials Science, Vietnam Academy of Science and Technology, Hanoi, Vietnam \\ ${ }^{5}$ Center for Innovative Materials and Architectures (INOMAR), Ho Chi Minh City, Vietnam \\ ${ }^{6}$ Faculty of Environmental and Chemical Engineering, Duy Tan University, Da Nang 550000, Vietnam \\ ${ }^{7}$ Future Materials and Devices Laboratory, Duy Tan University, Ho Chi Minh City 700000, Vietnam
}

Correspondence should be addressed to Viet-Duc Phung; phungvietduc2021@gmail.com and Nhu Hoa Thi Tran; ttnhoa@hcmus.edu.vn

Received 1 February 2021; Revised 22 February 2021; Accepted 5 March 2021; Published 18 March 2021

Academic Editor: Nguyen Duc Cuong

Copyright ( $2021 \mathrm{Vu}$ Thi Huong et al. This is an open access article distributed under the Creative Commons Attribution License, which permits unrestricted use, distribution, and reproduction in any medium, provided the original work is properly cited.

\begin{abstract}
Gold nanoparticles (Au NPs) were almost chosen as the first option for biological and biosensor applications due to their enhancement and their outstanding properties. The combining of optical fiber with localized surface plasmon resonance (LSPR) for forming a biosensor is widely used in diagnosis. In this work, we report a fiber optical biosensor based on LSPR of Au NPs for the detection of bovine serum albumin (BSA) protein. BSA was functionalized on Au NPs immobilized fiber optic sensing head (length of $1 \mathrm{~cm}$ ) via methanesulfonic acid (MSA) by carboxylic binding. It is the binding between the analytes with the surface-modified Au NPs that caused refractive index changes in the sensing medium led to changes in optical power at the output of the sensor. The detection limit of the LSPR fiber biosensor was found to be $0.18 \mathrm{ng} / \mathrm{mL}$ for the BSA detection with the low coefficient of variation $(\mathrm{CV})$ at under $1 \%$. We have demonstrated the effectiveness of combining multimode fiber with Au NPs to generate the biosensor as the label-free sensor that can be a feasible tool for highly sensitive, rapid response time, stable, and miniaturized point-of-care analytical systems.
\end{abstract}

\section{Introduction}

Gold nanoparticles (Au NPs) with unique properties such as excellent compatibility, intense light scattering/absorption, high surface area to volume ratios, highly selective interoperability through electrostatic interaction, stable structure, and nontoxic have become the first choice among plasmonic nanoparticles for biological and biosensor applications [13]. Au NPs also have a special phenome that is localized surface plasmon resonance (LSPR), which was widely studied recently in sensing platforms due to its great advantages [46]. It provides compact, label-free, highly sensitive, and stable biosensing for the detection of biological molecules $[7,8]$. LSPR is a phenomenon in metallic nanostructures related to the resonance of free-electron waves in a metal. The incident light could be in resonance with the oscillations of the surface electron at an excitation frequency, resulting in the collective oscillation of the surface plasmons, and it is called an LSPR mode [9].

It is due to the unique optical properties and surface chemistries that Au NPs were used as the promising nanomaterial in numerous different types of sensors such as surface-enhanced Raman scattering (SERS), fluorescence, electrochemical, and fiber optical-based LSPR sensor. 
Therein, the combining of optical fiber with the LSPR phenomenon helps to enhance the light-matter interaction that provided a sensor type with enhanced sensitivity, fast response, higher stability, more affordable, compact size, and lower limit of detect sensor than that others $[10,11]$. The basic principle of this sensor relies on the altar reflective index of ambient dielectrics around the sensing region and does not require the labeling of the target molecules.

In this work, Au NPs were synthesized via the seedmediated growth method for the fabrication of the LSPRbased optical biosensor for the detection of a standard protein in biosensor experiments that is bovine serum albumin (BSA). Mercaptosuccinic acid (MSA) with carboxylic acid $(-\mathrm{COOH})$ groups was modified on the sensing surface via $\mathrm{Au}$ NP thiol bond and was utilized for the immobilization of BSA. The properties of Au NPs were tracked through the spectroscopic measurements including UV-visible spectroscopy, powder X-ray diffraction (PXRD), and field emission scanning electron microscope (FESEM). Moreover, Fouriertransform infrared spectroscopy (FTIR) and water contact angles (WCAs) were used to confirm the presence of surface functional groups. We achieved a low limit of detection (LOD) of $0.18 \mathrm{ng} / \mathrm{mL}$ for BSA detection. This result in this study can be compared to be 5284 times better than Peng et al.'s work [12] about optical biosensors based on surface plasmon polaritons for detecting BSA and 20 times better than that Tran et al.'s [13] about optical fiber biochemical sensors. This result proved the effective combining between the optical properties of $\mathrm{Au}$ NPs and optical devices, unfolded potentials in biological analysis, and biosensor application.

\section{Experimental Section}

2.1. Materials and Reagents. Gold(III) chloride trihydrate $\left(\mathrm{HAuCl}_{4} \cdot 3 \mathrm{H}_{2} \mathrm{O}, 99.9 \%\right)$, (3-aminopropyl)triethoxysilane (APTES, 99\%), Mercaptosuccinic acid (MSA, 97\%), sodium citrate tribasic dihydrate $\left(\mathrm{HOC}(\mathrm{COONa})\left(\mathrm{CH}_{2} \mathrm{COO}-\right.\right.$ $\mathrm{Na})_{2} \cdot 2 \mathrm{H}_{2} \mathrm{O}, \mathrm{Na}_{3} \mathrm{Ctr}, 99 \%$ ), phosphate-buffered saline (PBS), and bovine serum albumin (BSA) were all supplied by Sigma-Aldrich Co., MO, USA. Ethanol $\left(\mathrm{C}_{2} \mathrm{H}_{5} \mathrm{OH}, 99.8 \%\right)$ was obtained from Fisher Ltd. (UK), and glycerol $\left(\mathrm{C}_{3} \mathrm{H}_{8} \mathrm{O}_{3}\right.$, 99\%) was provided by Duksan Pure Chemicals Co. (Ltd., Korea). Sodium hydroxide $(\mathrm{NaOH}, 96 \%)$ was purchased from Guangdong Guanghua Sci-Tech Co., Ltd. (China).

2.2. Synthesis of Gold Nanoparticles. Gold nanoparticles were synthesized by the seed-mediated growth method utilizing citrate reduction of gold(III) chloride trihydrate. Deionized (DI) water was used for all preparations. The seed nanoparticles were made by adding $\mathrm{Na}_{3} \mathrm{Ctr}$ rapidly into a round bottom storage flask containing the mixture of $200 \mu \mathrm{L}$ of $\mathrm{NaOH} 1 \mathrm{M}$ and $100 \mathrm{~mL}$ of $\mathrm{HAuCl}_{4} 1 \mathrm{mM}$ at $100^{\circ} \mathrm{C}$ under vigorous stirring. The solution was stirred stably for 15 minutes and stored at $4{ }^{\circ} \mathrm{C}$. Then, the resulting Au seeds were grown by adding $3.377 \mathrm{~mL}$ seed into $36.22 \mathrm{~mL}$ DI water, $0.176 \mathrm{~mL} \mathrm{Na} \mathrm{Natr}_{3}$, and $0.227 \mathrm{~mL}$ of $\mathrm{HAuCl}_{4} \cdot 3 \mathrm{H}_{2} \mathrm{O} 1 \mathrm{mM}$. The reaction was conducted in an ultrasonic bath at $\mathrm{RT}$ for
40 minutes. The final product was preserved in glass vials and stored at $4^{\circ} \mathrm{C}$ for further use.

2.3. Surface Modification of Fiber Core for BSA Detection. Multimode optical fibers have been used for the construction of this type of sensor for a number of reasons as follows. It is suitable for a wide variety of luminescent sources so it can be expanded for more applications. The larger core makes it easier to denature the surface for sensor application. It is easier to adjust and introduce light to the multimode fiber so it is easier to experiment.

The clad-free fiber (Multimode, NA 0.37, JFTLHPolymicro Technologies) with the length of $1 \mathrm{~cm}$ core sensing has been fabricated by removing part middle of the plastic buffer layer with a soldering machine (ATC-2450-III, ARIM, Korea), and the cladding layer with a mixture of acetone and ethanol $(3: 1)$. After removing, the silica $\left(\mathrm{SiO}_{2}\right)$ surface of the sensor head was modified with $\mathrm{Au}$ NPs for the LSPR sensor; then, the carboxylic groups were created on it for BSA detection as shown in Figure 1. Briefly, the fiber core surface was silanized using 3\% APTES after the generation of the hydroxyl groups $(-\mathrm{OH})$ on it by an oxygen plasma machine (CUTE-1MPR, Femto Science Inc., Korea) for 2 min. Subsequently, Au NPs were immobilized on the amine-modified sample via the strong electrostatic force. The immersing time for Au NPs to be coated on the surface was investigated $(4,8,12$, and $16 \mathrm{hr})$. Finally, the clad-free fiber was carboxyl-functionalized using MSA acid solution of $0.1 \mathrm{mM}$ in ethanol for $16 \mathrm{hr}$ at RT, then washed with methanol several times.

2.4. Optical Setup for Biosensor Measurements. Microfluidic fabrication for real-time optical biosensor measurement was proposed and implemented. Briefly, this device is constructed from the bonding PDMS (polydimethylsiloxane, Sylgard 184, Dow Corning Co., USA) mold and clean glass together as soon as they are treated by an oxidation plasma system with the installation of a clad-free fiber inside. The PDMS mold with two ports (inlet/outlet, a diameter of $1 \mathrm{~mm}$ ) and a straight-shaped flow channel are generated from pouring the mixture of cross-linker curing agent and silicone elastomer base $(v / v$, a ratio of $1: 10)$ into a mold and dried at $70^{\circ} \mathrm{C}$ during 30 minutes in an oven.

Figure 2 shows the optical measurement system including a $5 \mathrm{~mW}$ He-Ne laser (632.8 nm, LASOS LGK 7628), a coupling system (NA of 0.4 ), an aperture collimator mount (AD9.5F), a digital handheld optical power (PM 100D, Thorlabs, Newton, NJ, USA), and the peristaltic pump (Eleya, SMP-21, Japan) to inject the analyte solution into the channel of microfluidic. Two ports for injecting liquids into the channel were made from the plastic tubing (Eleya, Japan) with inner diameter : outer diameter $=1.15: 3.2 \mathrm{~mm}$.

2.5. Measurement Techniques. The gold nanoparticles were characterized utilizing UV-visible spectroscopy (V-730 visible/NIR, JASCO, Tokyo, Japan), powder X-ray diffraction (PXRD, Bruker D8 Advance diffractometer, $\lambda=1.54178 \AA$ ), and field emission scanning electron microscope (FESEM, Hitachi S4800, USA). The presence of the surface functional 

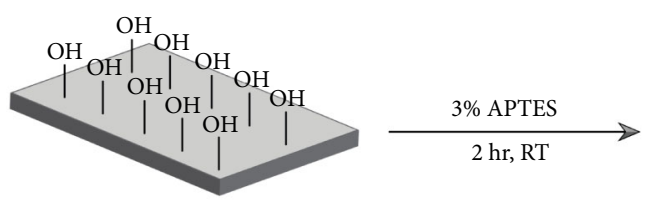

$\mathrm{O}_{2}$-treated $\mathrm{SiO}_{2}$

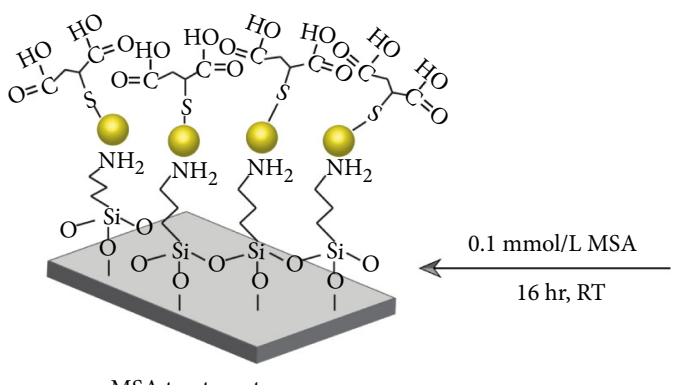

MSA treatment

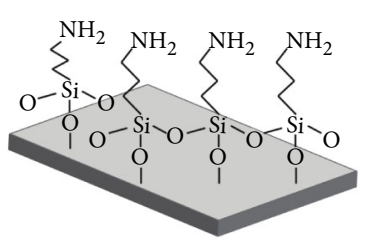

APTES treatment

$$
\downarrow \begin{gathered}
00 \\
\text { Au NPs } \\
\text { immersion }
\end{gathered}
$$

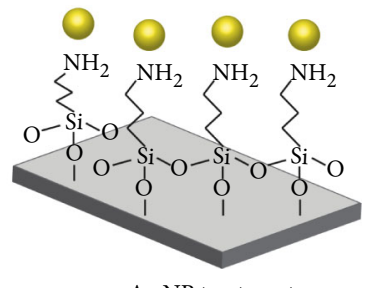

Au NP treatment

FIGURE 1: The illustrations of the surface modification steps for BSA detection.

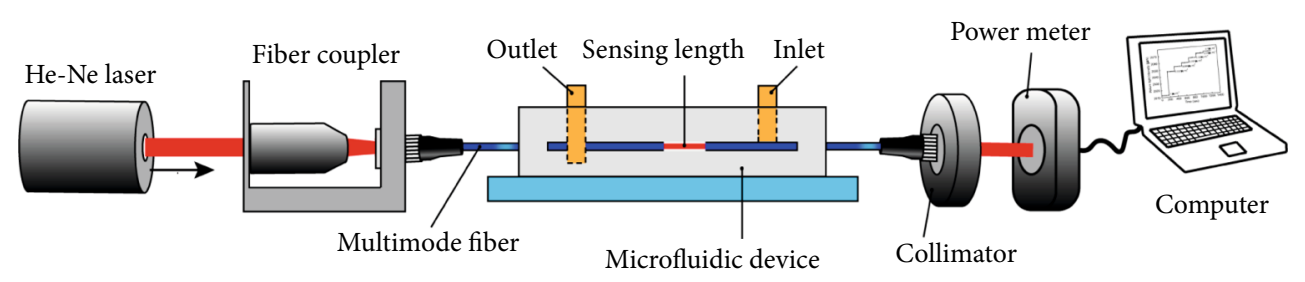

Figure 2: Diagram of the optical measurement system.

groups such as hydroxyl, carboxyl, and amino was investigated by the Fourier transform infrared spectroscopy (FTIR, Bruker Vertex 70, Germany) and water contact angles (WCAs, Phoenix 300, Surface Electro-Optics).

\section{Results and Discussion}

$\mathrm{Au}$ NPs were prepared by the seed-mediated growth method with the reduction of $\mathrm{HAuCl}_{4}$ utilizing $\mathrm{Na}_{3} \mathrm{Ctr}$ as a reducing agent. As soon as the synthesis of Au NPs is completed, the change in wavelength value and the color between Au nanoseed and Au NP solution was observed, shown in Figure 3. Briefly, a wine red color was obtained with the LSPR peak at $522.6 \mathrm{~nm}$ for the $\mathrm{Au}$ nanoseed solution. There are some disadvantages to the seed solution, such as uneven seed size and agglutination between seeds. The use of the intermediate particle development method helps to control the properties of the gold nanoparticles such as absorption size and wavelength, in addition to helping to produce single dispersed Au NPs. As the resulting Au seeds were grown, a slight redshift of the maximum absorbance peak from $522.6 \mathrm{~nm}$ to $524.5 \mathrm{~nm}$ with the change color to pink was recorded. The $\mathrm{Au}$ NPs formation with LSPR of $524.5 \mathrm{~nm}$ and the color of solution (Figure 3 ) implied the appearance of the spherical $\mathrm{Au}$ NPs, which is directed in this experiment due to the usefulness of them in bioapplications [14].

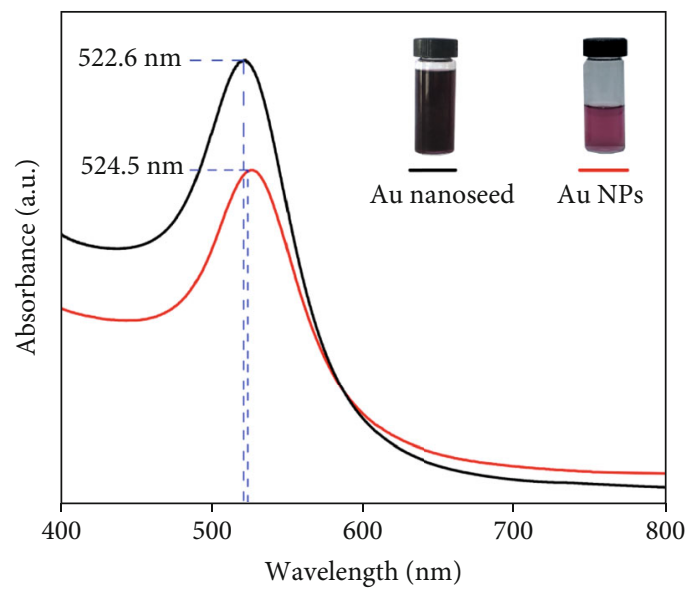

FIgURE 3: Wavelength shift in the UV-vis spectrum after the growth of Au NPs from Au nanoseed.

The proof of the presence of the characteristic functional group vibrations after the surface modification steps was recorded via FTIR spectroscopy and WCAs goniometry, as shown in Figure 4. In the FTIR spectrum (Figure 4(a)), the adsorption band at 450,687 , and $1140 \mathrm{~cm}^{-1}$ is attributed to Si-O-Si bending $(\delta)$, symmetric O-Si-O stretching $(v)$, and asymmetric Si-O-Si stretching $\left(v_{\text {as }}\right)$ vibrational, respectively [15]. The broadening in a band at 3370 to $3500 \mathrm{~cm}^{-1}$ 


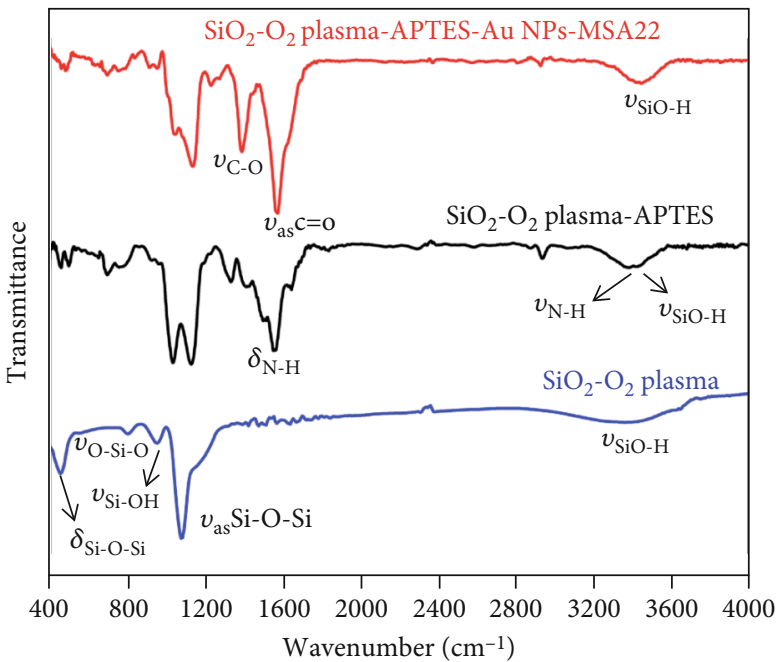

(a)

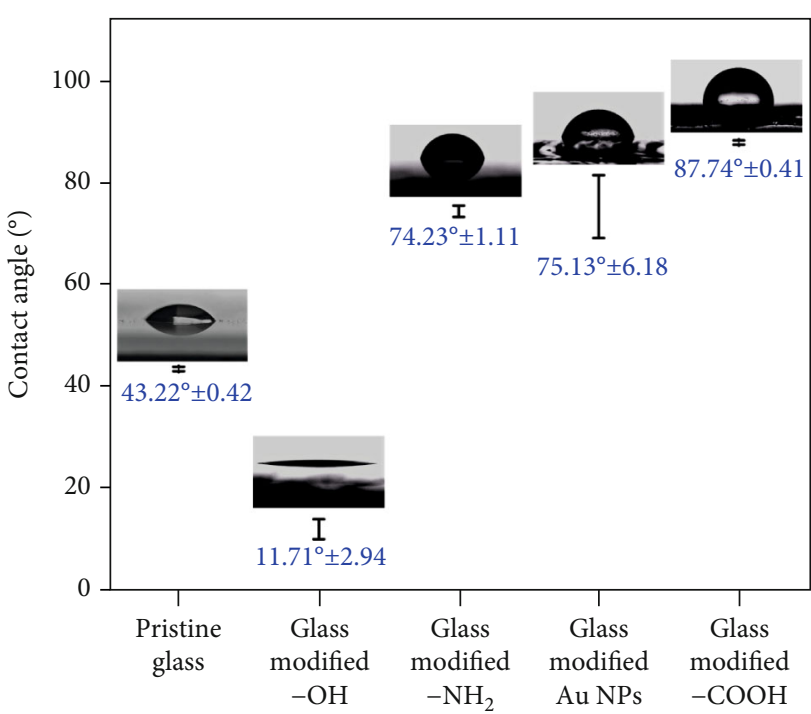

(b)

FIGURE 4: (a) FTIR spectra and (b) WCA goniometry of the modified silica surfaces.

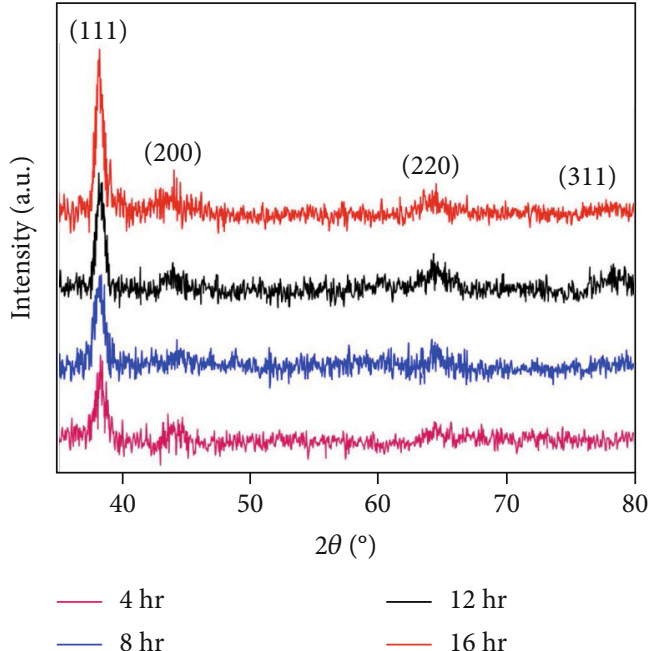

(a)
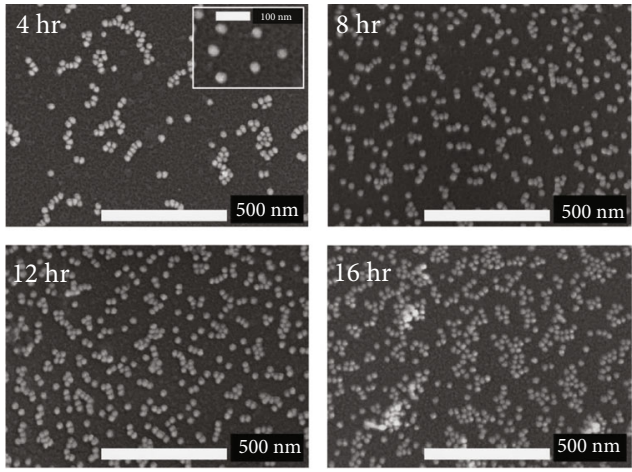

(b)

Figure 5: (a) XRD spectra and (b) FESEM image of the sample at the various gold immersing times $(4,8,12$, and $16 \mathrm{hr})$.

indicated the signal of -OH groups of the $\mathrm{Si}-\mathrm{OH}$ stretching vibration and retained water [16]. When modified with APTES, the IR spectrum was changed with the appearance of two peaks that is the N-H stretching and $\mathrm{N}-\mathrm{H}$ bending vibrations at 3350 and $1570 \mathrm{~cm}^{-1}$ [17]. In the spectrum of Au NP-MSA treatment, the amine vibration disappeared and the emergence of new bands at 1603 and $1340 \mathrm{~cm}^{-1}$ is assigned to asymmetric $\mathrm{C}=\mathrm{O}$ stretching and the $\mathrm{C}-\mathrm{O}$ stretching vibration, respectively [18]. In the results of WCA measurement (Figure 4(b)), the wetting behavior of the modified surfaces (the hydrophilic or hydrophobic) was investigated. Briefly, oxygen plasma-treated pristine glass indicated hydrophilicity due to a decrease from the angle of $43.22^{\circ} \pm 0.42$ to $11.71^{\circ} \pm 2.94$. After APTES functionalization, the wetting angle was increased to $74.23^{\circ} \pm 1.11$, imply- ing the hydrophobic layer on the surface. Then, Au NPcoated glass was determined to be more hydrophobic than the amine-modified surface with an angle of $75.13^{\circ} \pm 6.18$. After modification of Au NP-treated surface with MSA acid, the contact angle was increased sharply to $87.74^{\circ} \pm 0.41$, shown to turn super hydrophobic of the surface. The presence of the characteristic functional group vibrations in FTIR results and the surface wetting change in WCAs has indicated that $-\mathrm{OH},-\mathrm{NH}_{2}$, and $-\mathrm{COOH}$ groups were successfully formed on the wafer surface.

The properties of Au NPs including the shape, size, crystal structure were investigated via XRD spectra and FESEM image, as shown in Figure 5. The results show that the gold crystallines on the sample surface have the face-centered cubic (fcc) structure with (111), (200), (220), and (311) 


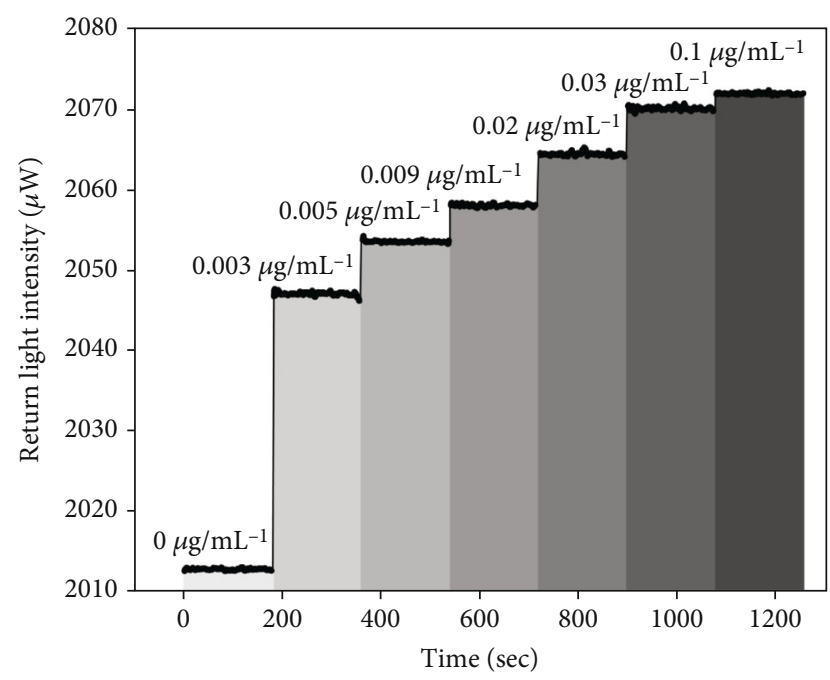

Figure 6: Time-dependent return light intensity via the optical fiber with the change of BSA concentration (from 0 to $0.1 \mu \mathrm{g} / \mathrm{mL}$ ).

planes corresponding to 2-theta values of $38.2,44.4,64.5$, and $77.4^{\circ}$ at the different immersing time $(4,8,12$ and $16 \mathrm{hr})$ (Figure 5(a)) [19]. The (111) plane is the strongest intensity peak indicated that it is the preferred development orientation of Au NPs on the substrate. Furthermore, the shape of $\mathrm{Au}$ NPs was displayed as monodispersity spherical nanoparticles with an average diameter of $32 \mathrm{~nm} \pm 3$ (Figure 5(b)). Increased immersion time led to an increase in particle count and the dispersion of Au NPs on the surface. According to the investigated results in Figures $5,12 \mathrm{hr}$ is considered as the appropriate candidate for use in subsequent experiments.

In order to evaluate the optical fiber-based LSPR sensor's performance, BSA was prepared in a list of various concentrations from 0 to $0.1 \mu \mathrm{g} / \mathrm{mL}$ in PBS buffer solution. The BSA solution was injected into the channel of microfluidic where the sensor head was contained. It was the addition of the analyte molecule that caused a change of refractive index (RI) of the sensor medium, which led to an increase or decrease in the light intensity at the output. The basic principle of this sensor is based on the refractive index change of the sensor medium, so it can detect different proteins with different refractive indexes and give different signals. BSA molecules were easily immobilized on the Au NP-covered sensing surface via $\mathrm{COOH}$ groups. The refractive index (RI) was altered in the direction of increase by the increasing of BSA concentration, proved in the work of Tran et al. [13]. To determine the LOD of our optical sensor, we measured and recorded the transmittance light in output at various RI of the ambient medium with fixed input light value. The results are presented in Figure 6 showing the sequential increase response of the sensor as the concentration of BSA was increased. The average signal intensity with the error bar at each concentration was recorded for $1 \mathrm{~min}$ and was repeated at least 3 times. Then, the coefficient of variation (CV) was estimated to be $<1 \%$, indicating the high reproducibility. This sensor was rated highly sensitive due to the signal that varies significantly and immediately as analyte concen-
TABLE 1: The performance of the sensor for detecting BSA.

\begin{tabular}{lcc}
\hline Biosensor methods & $\begin{array}{c}\text { Detection } \\
\text { limit }\end{array}$ & Ref. \\
\hline ELISA & $0.38 \mathrm{ng} / \mathrm{mL}$ & {$[20]$} \\
Vis-IR spectral & $0.05 \mathrm{mg} / \mathrm{mL}$ & {$[21]$} \\
$\begin{array}{l}\text { Reverse-phase high performance liquid } \\
\text { chromatography (RP-HPLC) }\end{array}$ & $0.11 \mu \mathrm{g} / \mathrm{mL}$ & {$[22]$} \\
$\begin{array}{l}\text { Two-dimensional transition metal } \\
\text { dichalcogenides assisted optical fiber SPR }\end{array}$ & $0.45 \mu \mathrm{g} / \mathrm{mL}$ & {$[23]$} \\
biosensor & & \\
SPR sensor probe without optical fibers & $50 \mathrm{ng} / \mathrm{mL}$ & {$[24]$} \\
This study & $0.18 \mathrm{ng} / \mathrm{mL}$ & \\
\hline
\end{tabular}

tration changes. The LOD value $(\mathrm{ng} / \mathrm{mL})$ of this sensor was calculated as 0.18 . This LOD is comparable to that of other sensors as shown in Table 1 indicating the high efficiency of the fiber optical LSPR biosensor. Our sensor therefore with this performance can be recognized as a feasible tool to be applied to biosensing.

\section{Conclusion}

In this article, an optical real-time measurement system and a biosensor device that contain the Au NP-coated fiber sensor head have been fabricated for sensor application. Au NPs have been synthesized by the seed-mediated growth method with the investigation of seed development through ultrasonic energy along with time change to find out the optimization in the properties of nanoparticles. Moreover, the process of surface denaturation of the sensor head of fiber optics with the modification of hydroxyl, amine, Au NPs, and carboxylic, respectively, for the analyte detection has been proposed. The combination of optical properties, X-ray diffraction technique, UV-vis spectroscopy, IR, and FESEM was used to follow the formation of crystal growth, absorption plasmon peak, sizes, and shapes of Au NPs. The high LOD of the LSPR sensor achieved $0.18 \mathrm{ng} / \mathrm{mL}$ for detection of BSA protein with remarkably high reproducibility. This research presented an in situ biosensor with highly sensitive in a compact format suited to the point-of-care testing.

\section{Data Availability}

The data used to support the findings of this study are included within the article.

\section{Conflicts of Interest}

The authors declare that they have no conflicts of interest.

\section{Authors' Contributions}

Vu Thi Huong and Nguyen Tran Truc Phuong contributed equally to this work. 


\section{Acknowledgments}

This research is funded by the Vietnam National Foundation for Science and Technology Development (NAFOSTED) under grant number 103.03-2019.379. The authors would like to gratefully acknowledge the Vietnam National University Ho Chi Minh City, Center for Innovative Materials and Architectures (Laboratory for Optics and Sensing).

\section{References}

[1] S. Zeng, K. Yong, I. Roy, X.-Q. Dinh, X. Yu, and F. Luan, “A review on functionalized gold nanoparticles for biosensing applications," Plasmonics, vol. 6, no. 3, pp. 491-506, 2011.

[2] P. K. Jain, K. S. Lee, I. H. El-sayed, and M. A. El-sayed, "Calculated absorption and scattering properties of gold nanoparticles of different size, shape, and composition: applications in biological imaging and biomedicine," Journal of Physical Chemistry B, vol. 110, no. 14, pp. 7238-7248, 2006.

[3] K. Saha, S. Agasti, C. Kim, X. Li, and V. M. Rotello, "Gold Nanoparticles in Chemical and Biological Sensing," Chemical Reviews, vol. 112, no. 5, pp. 2739-2779, 2012.

[4] P. Q. T. Do, V. T. Huong, N. T. T. Phuong et al., "The highly sensitive determination of serotonin by using gold nanoparticles (Au NPs) with a localized surface plasmon resonance (LSPR) absorption wavelength in the visible region," RSC Advances, vol. 10, no. 51, pp. 30858-30869, 2020.

[5] L. Chau, Y. Lin, S. Cheng, and T. Lin, "Fiber-optic chemical and biochemical probes based on localized surface plasmon resonance," Sensors and Actuators B: Chemical, vol. 113, no. 1, pp. 100-105, 2006.

[6] J. R. L. Guerreiro, M. Frederiksen, V. E. Bochenkov, V. de Freitas, M. G. Ferreira Sales, and D. S. Sutherland, "Multifunctional biosensor based on localized surface plasmon resonance for monitoring small molecule-protein interaction," ACS Nano, vol. 8, no. 8, pp. 7958-7967, 2014.

[7] K. A. Willets and R. P. Van Duyne, "Localized surface plasmon resonance spectroscopy and sensing," Annual Review of Physical Chemistry, vol. 58, no. 1, pp. 267-297, 2007.

[8] Y. Wang, J. Zhou, and J. Li, "Construction of Plasmonic NanoBiosensor-Based Devices for Point-of-Care Testing," Small Methods, vol. 1, no. 11, article 1700197, 2017.

[9] S. Unser, I. Bruzas, J. He, and L. Sagle, "Localized surface plasmon resonance biosensing: current challenges and approaches," Sensors, vol. 15, no. 7, pp. 15684-15716, 2015.

[10] M. Lu, H. Zhu, C. G. Bazuin, W. Peng, and J. F. Masson, "Polymer-templated gold nanoparticles on optical fibers for enhanced-sensitivity localized surface plasmon resonance biosensors," ACS Sensors, vol. 4, no. 3, pp. 613-622, 2019.

[11] H. Jeong, N. Erdene, J. Park, D. Jeong, H. Lee, and S. Lee, "Real-time label-free immunoassay of interferon-gamma and prostate-specific antigen using a Fiber-Optic Localized Surface Plasmon Resonance sensor," Biosensors and Bioelectronics, vol. 39, no. 1, pp. 346-351, 2013.

[12] Y. Peng, Y. Zhao, X. G. Hu, and Y. Yang, "Optical fiber quantum biosensor based on surface plasmon polaritons for the label-free measurement of protein," Sensors and Actuators B: Chemical, vol. 316, article 128097, 2020.

[13] N. H. T. Tran, J. Kim, T. B. Phan, S. Khym, and H. Ju, "Labelfree optical biochemical sensors via liquid-cladding-induced modulation of waveguide modes," ACS Applied Materials \& Interfaces, vol. 9, no. 37, pp. 31478-31487, 2017.

[14] P. N. Njoki, I. S. Lim, D. Mott et al., "Size correlation of optical and spectroscopic properties for gold nanoparticles," Journal of Physical Chemistry C, vol. 111, no. 40, pp. 14664-14669, 2007.

[15] A. M. Dattelbaum, M. L. Amweg, J. D. Ruiz, L. E. Ecke, A. P. Shreve, and A. N. Parikh, "Surfactant removal and silica condensation during the photochemical calcination of thin film silica mesophases," Journal of Physical Chemistry B, vol. 109, no. 30, pp. 14551-14556, 2005.

[16] A. S. Khan, H. Khalid, Z. Sarfraz et al., "Vibrational spectroscopy of selective dental restorative materials," Applied Spectroscopy Reviews, vol. 52, no. 6, pp. 507-540, 2017.

[17] N. Majoul, S. Aouida, and B. Bessaïs, "Progress of porous silicon APTES-functionalization by FTIR investigations," Applied Surface Science, vol. 331, pp. 388-391, 2015.

[18] I. M. A. Viegas, B. S. Santos, A. Fontes, G. A. L. Pereira, and C. F. Pereira, "Multivariate optimization of optical properties of CdSe quantum dots obtained by a facile one-pot aqueous synthesis," Inorganic Chemistry Frontiers, vol. 6, no. 6, pp. 1350-1360, 2019.

[19] M. Kamiński, K. Jurkiewicz, A. K. Burian, and A. Bródka, "The structure of gold nanoparticles: molecular dynamics modeling and its verification by X-ray diffraction," Journal of Applied Crystallography, vol. 53, no. 1, pp. 1-8, 2020.

[20] K. Zhang, C. Song, Q. Li, Y. Li, K. Yang, and B. Jin, “The establishment of a highly sensitive ELISA for detecting bovine serum albumin (BSA) based on a specific pair of monoclonal antibodies $(\mathrm{mAb})$ and its application in vaccine quality control," Human Vaccines \& Immunotherapeutics, vol. 6, no. 8, pp. 652-658, 2010.

[21] D. Shajari, A. Bahari, and P. Gill, "Fast and simple detection of bovine serum albumin concentration by studying its interaction with gold nanorods," Colloids and Surfaces A: Physicochemical and Engineering Aspects, vol. 543, pp. 118-125, 2018.

[22] M. Umrethia, V. L. Kett, G. P. Andrews, R. K. Malcolm, and A. D. Woolfson, "Selection of an analytical method for evaluating bovine serum albumin concentrations in pharmaceutical polymeric formulations," Journal of Pharmaceutical and Biomedical Analysis, vol. 51, no. 5, pp. 1175-1179, 2010.

[23] S. Kaushik, U. K. Tiwari, A. Deep, and R. K. Sinha, "Twodimensional transition metal dichalcogenides assisted biofunctionalized optical fiber SPR biosensor for efficient and rapid detection of bovine serum albumin," Scientific Reports, vol. 9, no. 1, p. 6987, 2019.

[24] T. Akimoto, S. Wada, and I. Karube, "A surface plasmon resonance probe without optical fibers as a portable sensing device," Analytica Chimica Acta, vol. 610, no. 1, pp. 119-124, 2008. 